\title{
Photosensible spiropyrans used as thermal sensor
}

\begin{abstract}
Photosensor materials are used in large-scale in the industry. These sensors are often used in equipment or products in areas such as health indicators, quality of food products, heating of equipment in the oil and gas industry, by warning danger situations. Unfortunately these sensors are not stable at high temperature. In this way, hybrid organic/inorganic materials can be used to improve the stability of the photosensor materials. Thus, in this work we report and hybrid temperature sensor, applied in equipment with low access to directly monitoring temperature in several processes, by using 1,3,3-trimethylidolino-beta-naftoespiropyran (Spiropyran T0423) in the development of a hybrid thermochromic sensor that indicates visual temperature modifications. The results indicate good agreement with traditional sensor, as Pt 100, but with just chance colour evaluation.
\end{abstract}

Volume 3 Issue 5 - 2017

\author{
Aline Vieira De Souza,Alexsandra Valério, \\ Emanoelle Diz Acosta, Ricardo Machado \\ Universidade Federal De Santa Catarina, Brazil
}

Correspondence: Ricardo Machado, Universidade Federal De Santa Catarina, Campus Trindade, Florianópolis, ZIP Code 88040-990, Santa Catarina State, Brazil, Tel +55 48 372I-243I, Email ricardo.machado@ufsc.br

Received: November 16, 2017| Published: November 30, 2017

Keywords: photosensor, spiropyran, hybrid polymers, temperature sensor

Abbreviations: IR, infrared spectra; MC, merocyanine; SP, spiropyran; UV, ultraviolet radiation

\section{Introduction}

Hybrid organic-inorganic materials are constituted by the combination of organic and inorganics compounds with a broad range of application and are an alternative for the production of novel multifunctional materials. ${ }^{1}$ These materials may be incorporated into a polymeric matrix, in conjunction with photosensitive or thermochromic molecules. Photochromism or the photochromatic effect is responsible for changing the colour of a material when exposed to a radiation source (UV and IR Spectra). The process is reversible and occurs through the light exposition, heating or chemical oxidation. ${ }^{2}$ Raditoiu et al. $^{3}$ incorporated thermochromic materials in the silicon aiming at stabilizing the thermal cycle and provide protection against solar rays and/or heating to avoid the photodegradation. Several molecules have photosensitive with thermochromic proprieties, and in this work, Spiropyran molecule (1,3,3-Trimethylindolino- $\beta$-naphthopyrylospiran) will be explored (Figure 1). ${ }^{3}$ Spiropyran is a reversible photosensitive molecule which is transformed into a polar hydrophilic isomer, the structure may return to the original form after being heated up and/or exposed to ultraviolet radiation. ${ }^{2,4,5}$ Spiropyrans are adapted to two stable modes: (1) open ring state, called MC, and (2) closed ring state, called SP. ${ }^{4}$ In this process that corresponds to a photoreversible and thermic process, MC may return to the original closed SP form due to heating and irradiation. During the reversion stage, notable changes in the molecule structure and charge distribution occur. ${ }^{4,5}$

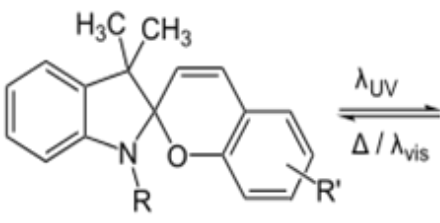

1

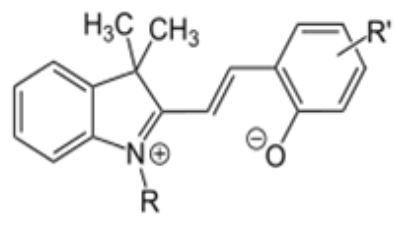

2
Figure I Chemical structure of spiropyran (I) and merocyanine (2). ${ }^{4}$

Photo and thermochromic spiropyrans molecules properties not only depend on the chemical nature of each of the compounds but also on the interface and synergy between them. ${ }^{6}$ Since charge transfer has a key role in the photochromism of these materials, being important to increase the charge (electrons and protons) and the interactions between both components. ${ }^{7}$ In other inorganic thermochromic materials, the colour change process involves an order-disorder transformation in the molecule at a specific temperature. ${ }^{8}$ A new area of study with extreme relevancy is the wireless sensors. Millions of sensors feed data in communication infra-structures, supplying information for health indications, food quality, menace or danger. ${ }^{9}$ However, some activities require traditional sensors, limiting the use of wireless sensors, because is necessary periodical calibration, associated with changes on the active detection surface of the device. ${ }^{10}$ These sensors are commonly used in the chemical industry, to humidity measurements and gas concentration, such as carbon dioxide. ${ }^{9}$ Other examples are the coloration change at the electrochemical detection of $\mathrm{pH}$, with difficulties in the implementation of wireless sensors. ${ }^{11,12}$

\section{Discussion}

One of the main limitations of the use of SP is the photodegradation regarding the sensorial system. Photosensitive materials are used in large scale on industries for the application of sensors and hybrid materials may interact improving its stability. These sensors are frequently used in equipment and products. ${ }^{13-15}$ The thermochromic and photosensitive sensor network may be applied for several areas as health indicators, food quality, heating of machines in industry and danger alarms. ${ }^{10}$ A large failing of this sensor occurs after the installation due to the constant calibration since changes occur on the sensor detection surface and may interfere with the measurement process. This process is complex and expensive and one of the solutions can be the use of thermochromic sensors. The most used photosensitive materials are the spiropyrans. ${ }^{16}$ This type of structure is not only activated by light and temperature but can be modulated to obtain several characteristics depending on the polymeric matrix used, as well as improved mechanical properties. However, the use of spiropyran has a limitation in the reversible detection, and the photodegradation is still an issue. ${ }^{6}$

\section{Conclusion}

A sensor with a photochromic or photosensitive response based on functional structures can be used for temperature monitoring. 
The sensor can be applied to equipment or devices exposed to high temperatures, which can be used for alerting, such as motors, heat exchangers, circuit breakers, transformers and notebook processors. These thermochromic or photosensitive sensors may be applied as health indicators and food quality reporting, by SOUZA (2014), where the sensor was applied in a device operating $170^{\circ} \mathrm{C}$ with good agreement if comprable to a conventional sensor, as Pt100.

\section{Acknowledgements}

The authors thank the financial support from ANP (Agência Nacional do Petróleo, Gás Natural e Biocombustíveis), Tintas Farben ${ }^{\circledR}$ and Programa de Recursos Humanos PRH 34 ANP/MCT.

\section{Conflict of interest}

The authors declares no conflict of interest.

\section{References}

1. Clément Sanchez, Beatriz Julián, Philippe Belleville, et al. Applications of hybrid organic-inorganic nanocomposites. Journal of Materials Chemistry. 205;15:3559-3592.

2. Berkovic G, Krongauz V, Weiss V. Spiropyrans and spirooxazines for memories and switches. Chem Rev. 2000;100(5):1741-1754.

3. Raditoiu V, Constantin R, Alina R, et al. Switching behavior of thermochromic copper and silver tetraiodomercurate embedded in silica hybrid materials. Optical Materials. 2013;35:2565-2572.

4. Souza AV, Valério A, Buske JLO, et al. Influence of stabilizer additives on thermochromic coating for temperature monitoring. Journal of Coatings Technology and Research. 2016;13(6):1139-1144.

5. Zhang W, Li X, Jiang Y, Yang R. Investigations of epoxy resins flameretarded by phenyl silsesquioxanes of cage and ladder structures. Polymer Degradation and Stability. 2013;98(1):246-254.
6. Radu A, Byrne R, Alhashimy N, et al. Spiropyran-based reversible, lightmodulated sensing with reduced photofatigue. Journal of Photochemistry and Photobiology A: Chemistry. 2009;206(2-3):109-115.

7. Lee A, Lichtenhan JD. Viscoelastic responses of polyhedral oligosilsesquioxane reinforced epoxy systems. Macromolecules. 1998;31(15):4970-4974.

8. Carotenuto G, Nicolais F. Reversible thermochromic nanocomposites based on thiolate-capped silver nanoparticles embedded in amorphous polystyrene. Materials. 2009;2(3):1323-1340.

9. Hairong QI, S Sitharam Iyengar, Krishnendu Chakrabarty. Distributed sensor networks-a review of recent research. Journal of the Franklin Institute. 2001;338(6):655-668.

10. Diamond D, Lau KT, Brady S, et al. Integration of analytical measurements and wireless communications current issues and future strategies. Talanta. 2008;75(3):606-612.

11. Kwang-Seok Yun, Joonho Gil, Jinbong Kim, et al. A miniaturized low-power wireless remote environmental monitoring system based on electrochemical analysis. Sensors and Actuators B: Chemical. 2004; 102(1):27-34.

12. Xiping Yang, Keat G Ong, William R Dreschel, et al. Design of a wireless sensor network for long-term, in-situ monitoring of an aqueous environment. Sensors. 2002;2(11):455-472.

13. Heilemann M, Dedecker P, Hofkens J, et al. Photoswitches: key molecules for subdiffraction-resolution fluorescence imaging and molecular quantification. Laser Photonics Reviews. 2009;3(1-2):180-202.

14. Feringa BL, Browne WR. Molecular Switches. 3rd ed. Germany: Wiley$\mathrm{VCH} ; 2011.792 \mathrm{p}$.

15. Byrne R, Diamond D. Chemo/bio-sensor networks. Nature Material. 2006;5(6):421-424.

16. Bakker E, Buehlmann P, Pretsch E. Carrier-based ion-selective electrodes and bulk optodes. 1. General characteristics. Chemical Reviews. 1997;97(8):3083-3132. 